\title{
Impact and acceptability of lay health trainer-led lifestyle interventions delivered in primary care: a mixed method study
}

\section{Shelina Visram}

Senior Lecturer, School of Medicine, Pharmacy and Health, Durham University, Queen's Campus, UK

\begin{abstract}
Aim: To evaluate the impact and acceptability of offering one-to-one lifestyle interventions delivered by lay health trainers in the primary care setting. Background: Chronic conditions represent major causes of ill-health, avoidable disability, pain and anxiety, and tend to be more prevalent in less affluent groups. This is due, in part, to the link between unhealthy lifestyles and lower socio-economic status, although factors such as poverty, worklessness and social exclusion play a larger role. Lay health trainers were introduced in England with the aim of providing personalised lifestyle advice, support and access to services for people living in disadvantaged areas. There is a body of literature on the effectiveness of lay or community health workers in the management of chronic conditions. However, little is known about their potential to promote lifestyle changes in newly diagnosed patients. An innovative health trainer service was piloted in the primary care setting, to work with people diagnosed with a chronic condition or identified as potentially benefitting from one-to-one support. Methods: A mixed method study design was utilised. Semi-structured interviews and focus groups were conducted with practice staff $(n=11)$ and patients $(n=15)$ from one primary care practice in North East England, United Kingdom. Discussions were audio-recorded and analysed using a thematic content approach. Routinely collected pre-/post-intervention data ( $n=246$ patients at baseline; sample sizes varied at end line) were analysed and appropriate descriptive and summary statistics produced. Findings: The discussions highlighted a high level of satisfaction with the health trainer model in terms of supporting positive lifestyle changes. Locating the intervention within the practice removed access barriers, particularly for those with long-term conditions. Anecdotal evidence of health improvement was supported by the quantitative analyses, which revealed statistically significant improvements in body mass index, blood pressure, dietary habits, exercise levels, alcohol intake, self-rated health and self-efficacy amongst those who completed the intervention.
\end{abstract}

Key words: health inequalities; health trainers; lifestyle; long-term conditions; mixed methods

Received 8 September 2016; revised 1 February 2017; accepted 7 February 2017; first published online 10 April 2017

\section{Introduction}

Lay health trainers were introduced in the United Kingdom with the aim of providing personalised advice, practical support and appropriate access to services for those living in socio-economically disadvantaged areas (Department of Health, 2004; Visram and South, 2013). They are often

Correspondence to: Dr Shelina Visram, Wolfson Building, School of Medicine, Pharmacy and Health, Durham University, Queen's Campus, Thornaby, Stockton-on-Tees, TS17 6BH, UK. Email: shelina.visram@durham.ac.uk

(C) Cambridge University Press 2017 
recruited from the target communities, thereby providing considerable potential for building community capacity and increasing involvement. Similar lay health workers (LHWs) have been used widely both in developed and developing countries. Previous reviews have identified positive effects from LHW programmes, often in terms of increasing access to care for disadvantaged and marginalised populations (Swider, 2002; Andrews et al., 2004). Specific areas where LHW interventions have been found to be effective and costeffective include: smoking cessation, tuberculosis treatment, HIV prevention, and education to reduce neonatal and maternal mortality (Islam et al., 2002; Sinanovic et al., 2003; Manandhar et al., 2004; Borghi et al., 2005; Carr et al., 2001; Pennington et al., 2013). There is also a separate but linked body of literature on the role of lay or peer support in the management of chronic conditions (Lorig et al., 1993; 1999; Reinschmidt et al., 2006). Studies report significant improvements in patient self-efficacy, general health and well-being, and self-care behaviour attributable to these interventions (Barlow et al., 2000; Griffiths et al., 2005; Kennedy, 2007; White et al., 2013; Bagnall et al., 2014).

Lay-led intervention models fit with the prevailing approach to long-term conditions in the United Kingdom, which involves a shift towards self-management and reducing demands on formal care. However, many people also have emotional or mental health problems that can reduce their ability and motivation to self-manage (Naylor et al., 2012). A recurring theme in the literature on health trainers involves the complex and challenging issues they face, as clients often present multiple physical, mental and social needs that are only revealed once a trusting relationship has been established (South et al., 2007; Dugdill et al., 2009; Ball and Nasr, 2011; Visram et al., 2014). Part of the health trainer role involves tackling social isolation by supporting people to become active in their communities, where possible. As such, health trainers form part of a wider system based on the ethos of 'care closer to home', which aims to avoid costly hospital treatment in the short-term and save money in the longer-term through prevention of avoidable illness and disability. A King's Fund report on clustering of unhealthy behaviours referred to health trainers as 'an under-used and ready-made workforce to help drive the reduction of multiple lifestyle risks' that tend to disproportionately affect those in less affluent areas (Buck and Frosini, 2012: 20).

A pilot health trainer project was developed in County Durham, northern England, to work with patients who were either newly diagnosed with a chronic condition or identified as potentially benefitting from support to make health-related lifestyle changes. Patients were also able to self-refer into the programme. The pilot ran for 21 months (from 1 December 2012 to 31 August 2014) and involved adult patients registered to a GP within Willington Medical Group receiving a one-to-one intervention for up to 12 weeks. The aims of this study were to evaluate the impact of the Willington Health Trainer Pilot (WHTP), and achieve insight into the acceptability of the innovative, health trainer-led approach.

\section{Method}

A mixed method study design was used, in order to qualitatively explore the range of experiences of those involved in the pilot and make best use of routinely collected monitoring data to test claims about its perceived impact.

\section{Participants}

Participants were recruited to the evaluation using a convenience sampling approach, which is commonly used in service development research (Robson, 2011). All practice staff were invited to take part, while having the option to decline without consequence. Information packs for patients who had completed the WHTP intervention by 31 March $2014(n=187)$ were prepared and address labels were added by National Health Service (NHS) administrative staff for distribution by post. The packs contained an invitation letter, information sheet, reply slip and pre-paid University envelope. On receipt of a completed reply slip, patients were contacted by the researcher and invited to take part in one of two focus groups. If they were unable to attend, they were also given the option to take part in a one-to-one interview.

WHTP patients were required to give written consent for their anonymised monitoring data to be used for evaluation purposes. Baseline data were therefore available for all patients who took part in the pilot programme, and end line data 
were available for those who attended a follow-up assessment on completion of the intervention.

\section{Procedure}

The intention was to conduct a single focus group with staff as part of a scheduled practice meeting, in order to gather their views and experiences of the WHTP. However, some were not able to take part in the group discussion (which involved six participants) and instead opted to participate in a paired interview $(n=2)$ or oneto-one interview $(n=3)$. These discussions took place either in a meeting room or private office within the practice, or by phone.

Patients were invited to take part in a focus group at a local community centre to discuss their experiences and perceptions of the programme. Two focus groups $\left(n_{1}=6\right.$ and $\left.n_{2}=4\right)$ were conducted, each lasting $\sim 90 \mathrm{~min}$. Participatory appraisal techniques, including ranking exercises and $\mathrm{H}$-diagrams, were used to generate discussion (Tock, 2001). The emphasis was on creating a relaxed, informal atmosphere, with a healthy lunch and refreshments provided by a local caterer. One patient chose to attend the same venue for an individual interview, while others $(n=4)$ opted to be interviewed by phone. All discussions were audio-recorded, with participants' consent and transcribed verbatim to assist with analysis.

Lifestyle questionnaires and biometric measurements were conducted by the health trainers with patients at baseline, and again on completion of the intervention, to capture any changes in body mass index (BMI), blood pressure, health-related behaviours, self-rated health (using a nonvalidated visual analogue scale, asking people to rate their general health from 1 to 10) and general self-efficacy score (a validated measure of the strength of a person's belief in their own ability to achieve their goals) (Chen et al., 2001). These data were recorded electronically using the national health trainer Data Collection and Reporting System (DCRS), before being anonymised and provided to the researcher for analysis.

\section{Analysis}

The focus group and interview transcripts were analysed using a thematic content approach, whereby each phrase is examined, coded according to the themes within it and considered in terms of its context in the discussion (Boyatzis, 1998). This process took place manually to ensure the researcher's continued immersion in the data. The analysis was then tested through discussions with the evaluation steering group, which included the practice managers and health trainer service managers. Ideas were also developed and tested during the patient focus groups; the use of ranking exercises enabled the participants to reach a consensus on important features of the WHTP intervention, which were then compared with data from the one-to-one patient interviews and discussions with staff (Tock, 2001). Monitoring data were analysed using the Statistical Package for the Social Sciences (SPSS) v.20. Descriptive statistics and repeated measures tests using appropriate parametric and non-parametric techniques were conducted to determine whether statistically significant changes were observed following completion of the intervention.

\section{Results}

In total, 255 patients were referred to the WHTP during the 21-month pilot period and, of these, 246 went on to commence the health trainer intervention. Others could not be contacted or chose not to continue for unknown reasons. The demographic characteristics of the WHTP patient population are summarised in Table 1. Two-thirds were female and half were aged between 46 and 65 years. The vast majority lived in more deprived areas, while less than $1 \%$ lived in the least deprived areas.

A total of 11 primary care staff and 15 patients took part in the focus groups or interviews (Tables 2 and 3). Three main themes emerged from the qualitative analysis: motivating factors; barriers and facilitators; and reported outcomes of the pilot. These themes are set out in turn below, illustrated by direct quotations from participants and supported by quantitative results where appropriate. Quotes are attributed using identifiers (S for staff and $\mathrm{P}$ for patients) to maintain anonymity.

\section{Motivating factors}

Most patients were referred to the programme by their GP or nurse for support relating to healthy eating and weight loss. Their motivation for 
Table 1 Willington Health Trainer Pilot patient population $(n=246)$

\begin{tabular}{llrr}
\hline Characteristic & Response & Number $\begin{array}{c}\text { Percentage } \\
(\%)\end{array}$ \\
\hline Gender & Female & 160 & 65.0 \\
& Male & 86 & 35.0 \\
Age group & Under 18 & 2 & 0.8 \\
& $18-25$ & 21 & 8.5 \\
& $26-35$ & 21 & 8.5 \\
& $35-45$ & 40 & 16.3 \\
& $46-55$ & 61 & 24.8 \\
& 56-65 & 59 & 24.3 \\
& 65+ & 37 & 15.0 \\
Ethnicity & Unknown & 5 & 2.0 \\
& White British & 216 & 87.8 \\
Deprivation & Not stated & 30 & 12.2 \\
quintile & Quintile 1 (most & 106 & 43.1 \\
& deprived) & & \\
& Quintile 2 & 111 & 45.1 \\
& Quintile 3 & 27 & 11.0 \\
& Quintile 4 & 2 & 0.9 \\
& Quintile 5 (most & 0 & 0 \\
& affluent) & & \\
\hline
\end{tabular}

Table 2 Staff focus group and interview sample $(n=11)$

\begin{tabular}{lll}
\hline Characteristic & Response & Number \\
\hline \multirow{2}{*}{ Gender } & Female & 10 \\
\multirow{2}{*}{ Role } & Male & 1 \\
& Receptionist or administrative & 5 \\
& assistant & 2 \\
& Practice manager & 2 \\
& Nurse or health-care assistant & 2 \\
& General practitioner & \\
\hline
\end{tabular}

Table 3 Patient focus group and interview sample $(n=15)$

\begin{tabular}{llc}
\hline Characteristic & Response & Number \\
\hline Gender & Female & 10 \\
Age group & Male & 5 \\
& Under 30 & 0 \\
& $30-39$ & 1 \\
& $40-49$ & 3 \\
& $50-59$ & 2 \\
& $60-69$ & 6 \\
Interventions status & $70-79$ & 3 \\
& $80+$ & 0 \\
& Completed & 10 \\
& Ongoing & 5 \\
\hline
\end{tabular}

Primary Health Care Research \& Development 2017; 18: 333-343 accessing the service was often linked to a desire to reduce or avoid symptoms associated with pre-existing long-term conditions:

[The doctor] asked me would I be interested and I said I'd be very interested. Because I was putting that much weight on, having arthritis in all of my body and my joints, I couldn't exercise properly. But since I've seen the health trainer and lost all this weight, it's brilliant. Absolutely brilliant. Best thing that's happened to me.

Referral by a health professional was felt to be an important factor by patients and staff, with the latter believing that the presence of an underlying medical problem represented 'an easy lead-in to offering [the intervention]' (S10) and contributed to a high completion rate $(92.3 \%)$. Staff described the pilot as being part of a triage system and contributing to continuity of care. Locating the health trainers in the practice was seen as key, particularly for those with chronic conditions:

It's one port of call. Supposing people have come to the diabetic clinic - what we're offering them is not something away on another site. It fits in with them coming perhaps for other things - blood pressure checks, blood tests. They can access it more easily. It's just making it less complex and less of a barrier, that they're coming to the same building where they'd access the other things that they need for their healthcare.

Some patients expressed a strong preference to participate in lifestyle interventions within their GP practice, while others simply desired a venue that was local and easy to access. The healthcare setting helped to formalise the intervention and some patients were more likely to attend for this reason:

It's going back to the same thing - that push. If you're going to see the doctor, in your head you're going to see the doctor. You're going to the doctors' premises aren't you, so it makes it more official. 


\section{Barriers and facilitators}

Two patients with multiple health complaints reported that participation in the WHTP intervention had produced no beneficial effects and felt that more specialist support was needed, particularly around accessing opportunities to be physically active:

The health trainer is fine, like I said, for people who are ill and able to do exercise and able to, you know... for I would say $99.9 \%$ of the population. But for people with long-term conditions and multiple conditions, it's too broad. It covers too broad a field.

Others were keen to receive ongoing support from the health trainers, either in the form of a drop-in facility or through occasional telephone contact. The fact that 'there wasn't really a support net there' (P6) was perceived to act as a potential barrier to maintaining any lifestyle changes.

On the whole, patients reported positive experiences of accessing and using the WHTP, in terms of feeling supported, receiving practical advice, and the non-judgemental, non-confrontational approach employed by the health trainers:

So relaxed! You know, before you know it you're telling him where you think you're going wrong. And he sort of said, 'Well you could try this'. It's not, 'You'd better try'. It's, 'Well, you could try...' And, 'Well, you haven't lost anything this week but if you try something you might lose it next week'. And that's a big difference to how you react to things, I think.

The emphasis was perceived to be on building confidence and empowering patients to change their own lifestyles, rather than telling them what to do or what not to do. It was recognised that health trainers have more time than other health professionals to explore patients' issues in-depth:

[The doctor] expects you to be as straight with him as he's being with you. And if you don't, if you tell him something he just takes it at face value, which is fine. Whereas [the health trainer] might... he's got that little bit longer and it's one-to-one and he can spend a little bit, he can probe around it a bit more, and that does help.

The use of practical resources and visual aids was seen as beneficial because 'it reinforced to you how much [weight] you'd actually lost, just visualising it like that made a big difference. And the same with the portion sizes' (P5). Staff described the health trainers as being knowledgeable about lifestyle issues and having the skills and resources needed to support their patients:

The health trainers are better at doing it [supporting lifestyle changes] than we are. They've got more time, they've got more practical resources in-house when they're seeing patients, and they can follow them up fairly regularly and give them the support that's needed. We don't have that luxury of time, and we're not as good as they are.

\section{Reported outcomes}

Patients reported making various lifestyle changes, including drinking more water, consuming fewer snacks and reducing their portion sizes. These changes were felt to have been relatively easy to make and to maintain following completion of the intervention. As a result, many had lost weight and some had managed to continue their weight loss:

I was nearly 16 stone and since I went to see [the health trainer] in September to this day I am 13 stone 10 [pounds]. And that's just by cutting portion sizes down. And I used to be a Coca Cola addict. I still crave it now but I used to drink cans after cans. But I haven't touched none since September.

I lost six stone, so I'm ready to do a knee replacement. That was the main reason I went and saw [the health trainer] and, with the help of my wife of course, changed the diet completely... She's lost two-and-a-half stone. 
The second quote above illustrates that there were occasions when the benefits of the service were felt to extend beyond individual patients, to "their social circles, their families and their friends' (S1). A number of patients reported tangible improvements in pre-existing conditions and staff had also observed 'very impressive health benefits' (S10) in patients:

I was [at the practice] on Monday - my blood pressure's great. I must have lost some blood when I had my knee replacement in August and I was anaemic, and then when I saw the nurse on Monday she said everything's fine. My blood pressure, it's gone down to 42, and she said I couldn't ask for anything better. So whatever I'm doing, I'm doing right.

With individuals I can think of who have got the approach, are motivated and stuck with it - fantastic improvements. A lady who got herself to a knee replacement much more comfortably with a much better surgical outcome than she would have done had she kept the amount of weight on that she had, was quite delighted with how far she'd come. Several other people who've made significant changes - mainly in weight loss - and improved their diabetic control.

Analysis of the pre- and post-intervention data highlighted significant improvements across all variables, including self-rated health and selfefficacy (Table 4). In total, 227 patients (92.3\%) achieved some or all of the goals set at the preintervention stage. These data provide evidence of health trainers successfully supporting individuals to make lifestyle changes, leading to improvements in objective (eg, BMI and blood pressure) as well as subjective measures (eg, self-rated health and self-efficacy).

Staff also reported observing improvements in patients' confidence levels. There was a sense that participation in the intervention could contribute to improved mental health and well-being, particularly for patients who were socially isolated:

Sometimes you need to talk to somebody. And as you say, it's not just your weight

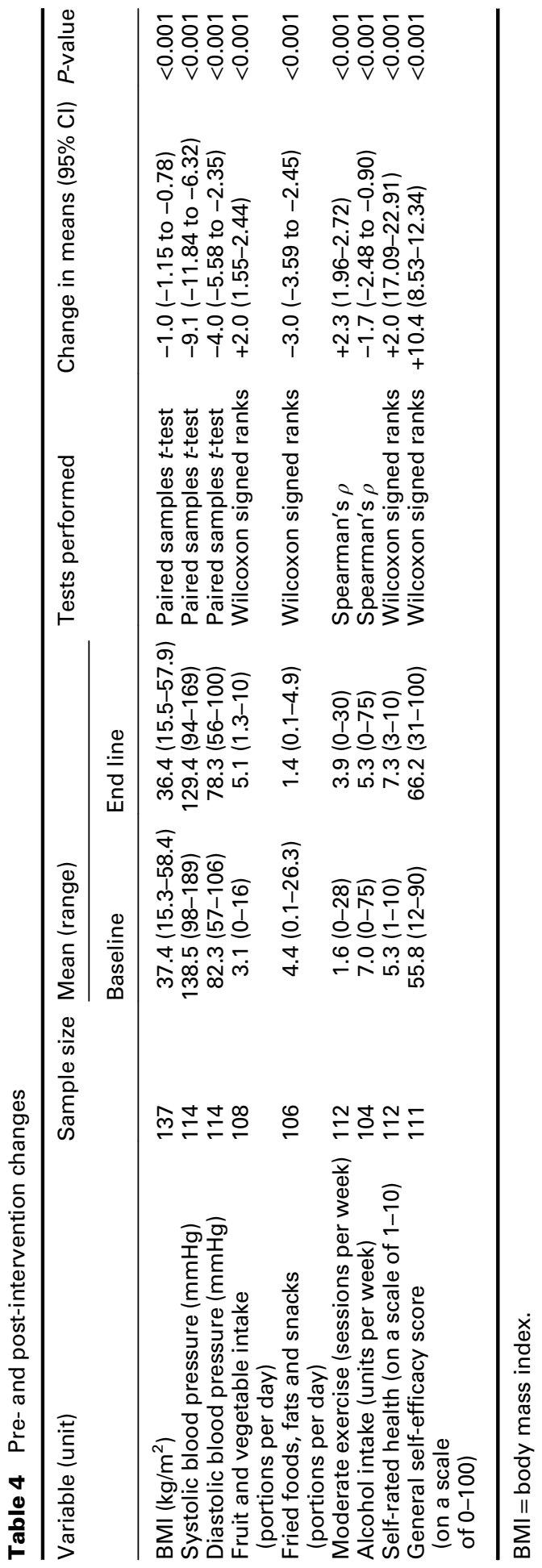


and that. It's your health and your mind. And if something's on your mind, you can talk to [the health trainer]. You feel trust and it makes you come away and you think I'm getting that off my chest, I feel great.

A final benefit concerned the perceived impact on the practice, in terms of a reduction in workloads for staff such as health-care assistants and diabetes specialist nurses:

It does lessen the amount of time I need to spend with patients in the diabetic clinic. Once I've referred them to the health trainer then they take a lot of that work away from me. So yeah, it could reduce the workload in that respect because I'm not having to go through all of their diet and things with them and give them the advice on losing weight.

\section{Discussion}

\section{Summary}

This evaluation indicates a high level of satisfaction with the WHTP approach in terms of supporting positive lifestyle changes amongst patients living in more deprived areas. Locating the intervention within the practice was felt to remove access barriers, particularly for those with longterm conditions. The health trainers were described as having the skills, knowledge and resources to provide tailored lifestyle advice, as well as having the time to work with patients on a one-to-one basis. Patients also appreciated the informal, non-judgemental approach. Anecdotal evidence of lifestyle change and health improvement was supported by analyses of the DCRS data. These results revealed statistically significant improvements in BMI, blood pressure, dietary habits, exercise levels, alcohol intake, self-rated health and self-efficacy following completion of the intervention. Primary care staff described the intervention as contributing to continuity of care, as well as a potential reduction in workloads.

\section{Strengths and limitations}

The findings presented here are based on a convenience sample of staff and patients from a single general practice, and the WHTP population largely involved white, female, middle-aged patients. As with any qualitative research, these findings solely reflect the views of the participants, which limits the ability to generalise to the wider population. Furthermore, the fieldwork and analyses were conducted by a single researcher, creating potential for bias. However, triangulation through use of a mixed methods approach and multiple study samples enhances the reliability of the evaluation (Denzin, 1978). Using extracts from participants' verbatim accounts also increases the dependability of the findings by grounding interpretations within the data (Johnson, 1997). Data were generated using different techniques appropriate to the study samples, with consideration given to the needs and preferences of each participant. Telephone interviews can be useful in geographically disperse populations, such as those living in more remote areas of County Durham, but the trade-off was the loss of additional insights arising from interactions between participants in a focus group setting (Robson, 2011).

The quantitative results were based on data gathered during routine practice, leading to concerns about possible recording errors and incomplete information. Collecting outcome data is known to be a challenge for health trainer services nationally (Mathers et al., 2017). The use of a nonvalidated measure of self-rated health and lack of a control group are additional weaknesses of the evaluation. Given that the pilot targeted those newly diagnosed with a chronic condition, it may be that the initial diagnosis and advice provided by their GP were the main factors in prompting patients to adopt healthier lifestyles. However, disadvantaged groups tend to be resistant to lifestyle advice from health professionals, as well as experiencing a lack of control over their circumstances and having a lower confidence and skill base (Lawlor et al., 2003; Michie et al., 2009). The WHTP was successful in working with patients from less affluent areas who were not likely to have made the observed behaviour changes without support. Individual level data were only available up to the end of the pilot and therefore the potential longer-term impact is unknown.

\section{Comparison with existing literature}

The evidence base relating specifically to health trainers is patchy but growing (Attree et al., 2011; Visram and South, 2013). Previous qualitative 
evaluations have highlighted the appeal of the oneto-one approach and having time to discuss healthrelated issues with an impartial but approachable stranger (South et al., 2007; Ball and Nasr, 2011; Visram et al., 2014). Holistic, flexible and tailored interventions are perceived as most successful, particularly those that combine practical and emotional support (Dugdill et al., 2009; Ward and Banks, 2009). Health trainer services tend to be better at engaging with women than men; analyses of national DCRS data for 2013-2014 found that $68.1 \%$ of clients were female, compared with $65.0 \%$ in the WHTP (Bagnall et al., 2014). It is well recorded that men are less likely to make use of health services than women, for reasons including caring being seen as a female task, work-related issues, and a lack of services specifically targeting men's health (Bertakis et al., 2000; Smith et al., 2006). Staff who took part in this evaluation were highly supportive of the WHTP, in contrast with literature suggesting that health professionals tend to be concerned that use of 'unregulated' workers might lead to reductions in the quality of care (Murphy, 2007).

Fewer studies have assessed the outcomes or cost-effectiveness of health trainer services. Only one definitive outcome evaluation has been conducted to date and found no evidence of the intervention having significant added impact, in terms of promoting lifestyle changes amongst patients with at least one risk factor for cardiovascular disease (Goodall et al., 2014). However, mean NHS and social service costs fell by slightly more in the intervention group, resulting in an incremental cost per quality adjusted life-year (QALY) of $£ 14480.34$ (Barton et al., 2012). This is more favourable than the threshold of $£ 20000$ per QALY gained set by the National Institute of Health and Care Excellence, suggesting that the intervention represented value for money. Limitations of this pilot randomised-controlled trial (RCT) include the small numbers involved (intervention group $n=76$, control group $n=38$ ) and the fact that many patients had no faceto-contact with a health trainer. Furthermore, most were from less deprived areas. Other studies have demonstrated that health trainers tend to be successful in reaching clients from lower socioeconomic groups and supporting them to achieve their behaviour change goals (Wilkinson et al., 2011; Bagnall et al., 2014; White et al., 2015).
Evaluations of similar lay-led programmes have demonstrated promising results. For example, a cluster RCT of the Cardiovascular Health Awareness Programme (CHAP) in Canada found significant reductions in hospital admissions for acute myocardial infarction and congestive heart failure in the intervention communities, equating to 3.02 fewer annual hospital admissions per 1000 people aged 65 and over (Kaczorowski et al., 2011). Successful implementation of CHAP in 20 intervention communities also demonstrated the feasibility and acceptability of this approach. Evidence to support the use of LHWs in contributing to reductions in BMI or improvements in mental health is mixed (Gary et al., 2003; Viswanathan et al., 2009). Increases in self-rated health, positive mood and psychological wellbeing have been found to be more likely in interventions seeking to improve chronic disease management, providing further support for the WHTP model (Carr et al., 2001). In a costeffectiveness analysis of the UK Expert Patients Programme, most of the intervention costs were offset by reductions in inpatient days, although these were largely attributable to a few resource-intensive patients (Richardson et al., 2002). Nevertheless, chronic disease management programmes offer the possibility of enabling patient empowerment at modest or zero overall cost (Pennington et al., 2013). In relation to management of type 2 diabetes or impaired glucose tolerance, studies consistently report falls in HbA1c levels attributable to the interventions (Gary et al., 2003; Young et al., 2005; Lujan et al., 2007; Betzlbacher et al., 2013). It is therefore possible to conclude that low intensity LHW interventions may be cost-effective at thresholds acceptable in the United Kingdom.

\section{Implications for practice}

Health trainer services appear to be highly acceptable to patients and primary care staff, as well as contributing to improvements in health and self-efficacy. The individualised, holistic intervention model was felt to be key to the success of the WHTP. Some patients expressed a preference to take part in interventions delivered within the GP practice, but most felt the personality and approach of the staff was more important than the provider. A hybrid model is likely to be most successful, involving a combination of activities delivered in community and primary care settings. Patients also 
expressed a need for ongoing support, particularly with their weight loss efforts. However, there are clear resource implications associated with providing a one-to-one intervention over the longerterm. The possibility of offering drop-in sessions and group-based activities should be explored, as well as providing follow-on support via telephone or text message where appropriate. Lay-led interventions also need to be situated within a wider public health system, with appropriate signposting and referral to specialist support.

\section{Acknowledgements}

The author expresses her thanks to the patients and staff who gave up their time to take part in the interviews and focus groups. The support and assistance of staff from County Durham and Darlington NHS Foundation Trust and Willington Medical Group in undertaking the study is also gratefully acknowledged.

\section{Financial Support}

This work was supported by a small grant from County Durham and Darlington NHS Foundation Trust.

\section{Conflicts of Interest}

None.

\section{Ethical Standards}

The author asserts that all procedures contributing to this work comply with ethical standards in NHS research, as well as the Data Protection Act and the Helsinki Declaration of 1975 , as revised in 2008. The protocol was approved by the School of Medicine, Pharmacy and Health Research Ethics Sub-Committee at Durham University (ref. ESC2/ 2013/PP010) and research governance approval was granted by County Durham and Darlington NHS Foundation Trust.

\section{References}

Andrews, J., Felton, G., Wewers, M. and Heath, J. 2004: Use of community workers in research with ethnic minority women. Journal of Nursing Scholarship 36, 358-65.
Attree, P., Clayton, S., Karunanithi, S., Nayak, S., Popay, J. and Read, D. 2011: NHS health trainers: a review of emerging evaluation evidence. Critical Public Health 22, 25-38.

Bagnall, A., Trigwell, J. and White, J. 2014: Health trainers end of year review 1st April 2013 - 31st March 2014. Leeds: Leeds Metropolitan University.

Ball, L. and Nasr, N. 2011: A qualitative exploration of a health trainer programme in two UK primary care trusts. Perspectives in Public Health 131, 24-31.

Barlow, J., Turner, A. and Wright, C. 2000: A randomized controlled study of the arthritis management programme in the UK. Health Education Research 15, 665-810.

Barton, G., Goodall, M., Bower, P., Woolf, S., Capewell, S. and Gabbay, M. 2012: Increasing heart-health lifestyles in deprived communities: economic evaluation of lay health trainers. Journal of Evaluation in Clinical Practice $18,835-40$.

Bertakis, K., Azari, R., Helms, J., Callahan, E. and Robbins, J. 2000: Gender differences in the utilization of health care services. Journal of Family Practice 49, 147-52.

Betzlbacher, A., Grady, K., Savas, L., Cotterill, S., Boaden, R., Summers, L. and Gibson, M. 2013: Behaviour change among people with impaired glucose tolerance: comparison of telephone-based and face-to-face advice. Journal of Health Service Research and Policy 18, 2-6.

Borghi, J., Thapa, B., Osrin, D., Jan, S., Morrison, J., Tamang, S., Shrestha, B.P., Wade, A., Manandhar, D.S. and de L Costello, A.M. 2005: Economic evaluation of a women's group intervention to improve birth outcomes in rural Nepal. The Lancet 366, 1882-884.

Boyatzis, R. 1998. Transforming qualitative information: thematic analysis and code development. London: Sage.

Buck, D. and Frosini, F. 2012: Clustering of unhealthy behaviours over time. Implications for policy and practice. London: The King's Fund.

Carr, S., Lhussier, M., Forster, N., Geddes, L., Deane, K., Pennington, M., Visram, S., White, M., Michie, S., Donaldson, C. and Hildreth, A. 2001: An evidence synthesis of qualitative and quantitative research on component intervention techniques, effectiveness, cost-effectiveness, equity and acceptability of different versions of healthrelated lifestyle advisor role in improving health. Health Technology Assessment 15, 1-300.

Chen, G., Gully, S. and Eden, D. 2001: Validation of a new general self-efficacy scale. Organizational Research Methods 4, 62-83.

Denzin, N.K. 1978. The Research Act: a theoretical introduction to sociological methods. New York: McGraw-Hill.

Department of Health 2004. Choosing health: making healthy choices easier. London: The Stationery Office.

Dugdill, L., Coffey, M., Coufopoulos, A., Byrne, K. and Porcellato, L. 2009: Developing new community health roles: can reflective learning drive professional practice? Reflective Practice 10, 121-30.

Gary, T., Bone, L., Hill, M., Levine, D., Mcguire, M., Saudek, C. and Brancati, F. 2003: Randomized controlled trial of the 
effects of nurse case manager and community health worker interventions on risk factors for diabetes-related complications in urban African Americans. Preventive Medicine 37, 23-32.

Goodall, M., Barton, G.R., Bower, P., Byrne, P., Cade, J.E., Capewell, S., Cleghorn, C. L., Kennedy, L.A., Martindale, A.M., Roberts, C., Woolf, S. and Gabbay, M.B. 2014: Food for thought: pilot randomized controlled trial of lay health trainers supporting dietary change to reduce cardiovascular disease in deprived communities. Journal of Public Health $36,635-43$.

Griffiths, C., Motlib, J., Azad, A., Ramsay, J., Eldridge, S., Feder, G. and Al, E. 2005: Randomised controlled trial of a lay-led self-management programme for Bangladeshi patients with chronic disease. British Journal of General Practice 55, 831-37.

Islam, M., Wakai, S., Ishikawa, N., Chowdhury, A. and Vaughan, J. 2002: Cost-effectiveness of community health workers in tuberculosis control in Bangladesh. Bulletin of the World Health Organisation 80, 445-50.

Johnson, R. 1997: Examining the validity structure of qualitative research. Education 118, 282-92.

Kaczorowski, J., Chambers, L., Dolovich, L., Paterson, J., Karwalajtys, T., Gierman, T., Farrell, B., Mcdonough, B., Thabane, L., Tu, K., Zagorski, B., Goeree, R., Levitt, C., Hogg, W., Laryea, S., Carter, M., Crosee, D. and Sebalt, R. 2011: Improving cardiovascular health at population level: 39 community cluster randomised trial of Cardiovascular Health Awareness Program (CHAP). British Medical Journal 342, d442.

Kennedy, A. 2007: The effectiveness and cost effectiveness of a national lay-led self care support programme for patients with long-term conditions: a pragmatic randomised controlled trial. Journal of Epidemiology and Community Health 61, 254-61.

Lawlor, D., Frankel, S., Shaw, M., Ebrahim, S. and DaveySmith, G. 2003: Smoking and ill-health: does lay epidemiology explain the failure of smoking cessation programs among deprived populations? American Journal of Public Health 93, 266-70.

Lorig, K., Sobel, D., Stewart, A., Brown, B., Bandura, A., Ritter, P., Gonzalez, V.M., Laurent, D.D. and Holman, H.R. 1999: Evidence suggesting that a chronic disease selfmanagement programe can improve health status while reducing hospitalization: a randomized trial. Medical Care $1,5-14$.

Lorig, K.R., Mazonson, P.D. and Holman, H.R. 1993: Evidence suggesting that health education for self-management in patients with chronic arthritis has sustained health benefits while reducing health care costs. Arthritis and Rheumatism 36, 439-46.

Lujan, J., Ostwald, S. and Ortiz, M. 2007: Promotora diabetes intervention for Mexican Americans. Diabetes Education $33,660-70$.

Manandhar, D., Osrin, D., Shrestha, B.P., Mesko, N., Morrison, J., Tumbahangphe, K.M., Tamang, S., Shrestha, D., Thapa, B., Shrestha, J.R., Wade, A., Borghi, J., Standing, H.,

Primary Health Care Research \& Development 2017; 18: 333-343
Manandhar, M. and Costello, A. 2004: Effect of a participatory intervention with women's groups on birth outcomes in Nepal: cluster-randomized controlled trial. The Lancet 364, 970-79.

Mathers, J., Taylor, R. and Parry, J. 2017: Measuring the impact of Health Trainers Services on health and health inequalities: does the service's data collection and reporting system provide reliable information? Journal of Public Health 39, 139-44.

Michie, S., Jochelson, K., Markham, W. and Bridle, C. 2009: Low-income groups and behaviour change interventions: a review of intervention content, effectiveness and theoretical frameworks. Journal of Epidemiology and Community Health 63, 610-22.

Murphy, B. 2007: Medical role substitutions and delegations overcoming the fear. Australian Health Review 31, S20-24.

Naylor, C., Parsonage, M., Mcdaid, D., Knapp, M., Fossey, M. and Galea, A. 2012. Long-term conditions and mental health. The cost of co-morbidities. London: The King's Fund.

Pennington, M., Visram, S., Donaldson, C., White, M., Lhussier, M., Deane, K., Forster, N. and Carr, S. 2013: Cost-effectiveness of health-related lifestyle advice delivered by peer or lay advisors: synthesis of evidence from a systematic review. Cost Effectivness and Resource Allocation 11, 30.

Reinschmidt, K., Hunter, J., Fernandez, M., Lacy-Martinez, C., Dezapien, J. and Meister, J. 2006: Understanding the success of promotoras in increasing chronic disease screening. Journal of Health Care for the Poor and Underserved 17, 256-64.

Richardson, G., Kennedy, A., Reeves, D., Bower, P., Lee, V., Middleton, E. and AL, E. 2002: Cost effectiveness of the Expert Patients Programme (EPP) for patients with chronic conditions. Journal of Epidemiology and Community Health 62, 361-67.

Robson, C. 2011. Real World Research: a resource for users of social research methods in applied settings. Oxford: Blackwell.

Sinanovic, E., Floyd, K., Dudley, L., Azevedo, V., Grant, R. and Maher, D. 2003: Cost and cost-effectiveness of communitybased care for tuberculosis in Cape Town, South Africa. International Journal of Tuberculosis and Lung Disease 7, S56-62.

Smith, J., Braunack-Mayer, A. and Wittert, G. 2006: What do we know about men's help-seeking and health service use? Medical Journal of Australia 184, 81-83.

South, J., Woodward, J. and Lowcock, D. 2007: New beginnings: stakeholder perspectives on the role of health trainers. Journal of the Royal Society for the Promotion of Health 127, 224-30.

Swider, S. 2002: Outcome effectiveness of community health workers: an integrative literature review. Public Health Nursing 19, 11-20.

Tock, L. 2001: Participatory appraisal: a brief introduction [Online]. London: shortwork. Retrieved 23 November 2016 from http://shortwork.org.uk/participatory-research/ an-introduction-to-participatory-appraisal/. 
Visram, S., Clarke, C. and White, M. 2014: Making and maintaining lifestyle changes with the support of a lay health advisor: longitudinal qualitative study of health trainer services in northern England. PLoS One 9, e94749.

Visram, S. and South, J. 2013: Guest editorial: building an evidence base for health trainers. Perspectives in Public Health 133, 193-94.

Viswanathan, M., Kraschnewski, J., Nishikawa, B., Morgan, L., Thieda, P., Honeycutt, A., Lohr, K. and Jonas, D. 2009: Outcomes of community health worker interventions. Evidence Report/Technology Assessment No. 181. Rockville, MD: Agency for Healthcare Research and Quality.

Ward, L. and Banks, L. 2009. Evaluation of health trainers in West Sussex. Brighton: Social Science Policy and Research Centre, University of Brighton.
White, J., Bagnall, A.-M. and Trigwell, J. 2015: Health trainers making a difference to mental health and wellbeing. Perspectives in Public Health 135, 130-32.

White, J., Woodward, J. and South, J. 2013: Addressing inequalities in health - what is the contribution of health trainers? Perspectives in Public Health 133, 213-20.

Wilkinson, D., Sniehotta, F. and Michie, S. 2011: Targeting those in need: baseline data from the first English National Health Service (NHS) Health Trainer Service. Psychology, Health \& Medicine 16, 36-48.

Young, R., Taylor, J., Friede, T., Hollis, S., Mason, J., Lee, P., Burns, E., Long, A., Gambling, T., New, J. and Gibson, J. 2005: Proactive call centre treatment support (PACCTS) to improve glucose control in type 2 diabetes. Diabetes Care 28, 278-82. 